\title{
Truncation of galaxy dark matter halos in high density environments
}

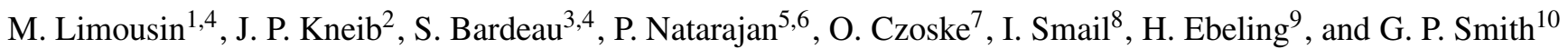 \\ 1 Dark Cosmology Centre, Niels Bohr Institute, University of Copenhagen, Juliane Maries Vej 30, 2100 Copenhagen, Denmark \\ e-mail: marceau@dark-cosmology.dk \\ 2 OAMP, Laboratoire d'Astrophysique de Marseille - UMR 6110, Traverse du siphon, 13012 Marseille, France \\ ${ }^{3}$ L3AB - UMR 5804, 2 rue de l'Observatoire, BP 89, 33270 Floirac, France \\ 4 Laboratoire d'Astrophysique de Toulouse-Tarbes, CNRS-UMR 5572 \& Université Paul Sabatier Toulouse III, \\ 14 avenue Édouard Belin, 31400 Toulouse, France \\ 5 Astronomy Department, Yale University, PO Box 208101, New Haven, CT 06520-8101, USA \\ 6 Department of Physics, Yale University, PO Box 208101, New Haven, CT 06520-8101, USA \\ 7 Argelander-Institut für Astronomie, Universität Bonn, Auf dem Hügel 71, 53121 Bonn, Germany \\ 8 Institute for Computational Cosmology, Durham University, South Road, Durham DH1 3LE, UK \\ 9 Institute for Astronomy, University of Hawaii, 2680 Woodlawn Drive, Honolulu, HI 96822, USA \\ 10 School of Physics and Astronomy, University of Birmingham, Edgbaston, Birmingham, B15 2TT, UK
}

Received 4 May 2006 / Accepted 10 October 2006

\section{ABSTRACT}

\begin{abstract}
Aims. Our aim is to constrain the properties of dark matter halos inhabiting high density environments, such as is the case in massive galaxy clusters.

Methods. We use galaxy-galaxy lensing techniques that utilize a maximum likelihood method to constrain the parameters of the lenses. It has been demonstrated that such a technique provides strong constraints on the parameters that characterize a galaxy halo, as well as on the aperture mass of these halos. In this analysis, we only use weak shear data and do not include strong lensing constraints. Results. We present the results of a study of galaxy-galaxy lensing in a homogeneous sample of massive X-ray luminous clusters at $z \sim 0.2$. These have been observed in three bands with the $\mathrm{CFH} 12 \mathrm{~K}$ instrument. We find dark matter halos in these clusters to be compact compared to those inferred around isolated field galaxies of equivalent luminosity at this redshift: the half mass radius is found to be smaller than $50 \mathrm{kpc}$, with a mean total mass of order $0.2 \times 10^{12} M_{\odot}$. This is in good agreement with previous galaxy-galaxy lensing results and with numerical simulations, in particular with the tidal stripping scenario. We thus provide a strong confirmation of tidal truncation from a homogeneous sample of galaxy clusters. Moreover, it is the first time that cluster galaxies are probed successfully using galaxy-galaxy lensing techniques from ground based data.
\end{abstract}

Key words. cosmology: dark mater - Galaxy: halo - gravitational lensing

\section{Introduction}

Gravitational lensing has become a very powerful tool to measure the mass distribution of structures in the Universe on a large range of scales. Both the strong lensing and the weak lensing regime are used to map mass distributions. On large scales, the weak distortion detected in the shapes of distant galaxies allows us to study the distribution of matter on cosmic scales (Refregier et al. 2003). On galaxy cluster scales, the strong lensing features observed in the cores of massive clusters can be used to put strong constraints on the inner part of the cluster potential (Kneib et al. 1996; Smith et al. 2005), whereas at larger clustercentric radius, in the weak lensing limit, the ellipticities of background galaxies give an estimate of the shear field induced by the gravitational potential of the foreground cluster. On the scale of individual galaxies, much work has been done as well on modeling and understanding multiple quasar systems (Fassnacht et al. 1999; Phillips et al. 2004). These strong galaxy-galaxy lensing analyses provide a test of structure formation in cold dark matter models since lensing provides an interesting way to estimate the inner slopes of density profiles, which can be compared to theoretical expectations.
Weak galaxy-galaxy lensing studies provide constraints on the physical parameters that characterize the dark matter halos of galaxies. This is accomplished directly using lensing, since the deformation in the shapes of background galaxies produced by the foreground lenses although weak is observationally detected statistically. Galaxy-galaxy lensing has been used to get constraints on field galaxies in different surveys (see Sect. 2 for a review). This effect has also been used successfully to map substructure in massive galaxy clusters (see Sect. 2.4), where most previous work has utilized strong and weak lensing features in order to constrain the properties of galaxy halos associated with the locations of bright early-type galaxies in clusters.

In this paper, we report the first detection of galaxy-galaxy lensing in clusters from ground based observations without utilizing strong lensing. We demonstrate that this technique works even without the constraints from multiple images but as a consequence we need more foreground-background pairs. To illustrate this we apply the technique to a well defined homogeneous sample of clusters all at $z \sim 0.2$. Additionally, using 3 photometric bands, we apply photometric redshift determination techniques to assign redshifts to all the background objects. The details of our method have been presented in a recent theoretical 
paper (Limousin et al. 2005, hereafter Paper I). It was demonstrated by analysing simulated data that the technique works well and allows us to retrieve the characteristic parameters that describe the dark matter halos and hence to put strong constraints on the aperture mass of these halos. We confirm the fact that galaxy halos in clusters are compact compared to halos of field galaxies of equivalent luminosity. This is a strong confirmation since it relies on a homogeneous sample of galaxy clusters, whereas most early work relied on a heterogeneous sample. The results presented here are averaged on a cluster galaxy population from the centre of the cluster up to $\sim 2 \mathrm{Mpc}$, which represent a significant fraction of the virial radius (depending on the cluster: for the sample we consider in this work, the virial radius spans from 2.5 Mpc for Abell 2218 to 3.6 Mpc for Abell 1835).

This paper is organized as follows: In Sect. 2 we review galaxy-galaxy lensing results to date; Sect. 3 describes the data and reduction techniques, as well as the procedure used to derive catalogues from these images. The determination of photometric redshifts is described in Sect. 4 and illustrated for the specific case of Abell 1763. The formalism used for modeling dark matter halos and the maximum likelihood method are outlined in Sect. 5. More details on the analysis are given in Paper I. Our results are presented in Sect. 6, where we also make comparisons with other galaxy-galaxy lensing results. Discussion of the results and conclusions are presented in Sect. 7. All our results in this paper are scaled to the flat, low matter density $\Lambda$ CDM cosmology with $\Omega_{M}=0.3, \Omega_{\Lambda}=0.7$ and a Hubble constant $H_{0}=65 \mathrm{~km} \mathrm{~s}^{-1} \mathrm{Mpc}^{-1}$. In such a cosmology, at $z=0.2,1^{\prime \prime}$ corresponds to $3.55 \mathrm{kpc}$.

\section{Galaxy-galaxy lensing as a probe of galaxy properties}

In this section, we provide a review of galaxy-galaxy lensing results to date: we begin by reviewing theoretical works and then summarize observational results.

\subsection{Theoretical analyses}

Galaxy-galaxy lensing methods have been developed just after the first detection of the phenomena (Brainerd et al. 1996, hereafter BBS). Different observational configurations were considered: for field galaxies, the work and techniques have been pioneered by Schneider \& Rix (1997) and in cluster by Natarajan \& Kneib (1997). Other theoretical works followed by Geiger \& Schneider (1998) and Limousin et al. (2005), respectively in cluster and both in cluster and in the field. Natarajan \& Kneib (1997) partitioned the mass of the cluster into a smooth clump and sub-halos that are associated with early-type galaxies. Robust constraints are obtained on both these components using strong and weak lensing observations. The presence of a few multiply imaged systems in the clusters that they modeled (with known measured redshifts) gave tighter error bars on the properties of the dark halos that they obtain. Other theoretical work does not include any strong lensing constraints in the analysis. The more recent study by Limousin et al. (2005) went beyond the usual formulation and proposed a re-parameterization of the problem in terms of more direct physical quantities that allows putting strong constraints on the aperture mass of a galaxy halo. These studies have demonstrated that it is possible to recover the input parameters of the lenses. They used a generated simulated catalogue defined to match present day observations in terms of shape parameter measurements and object number density. It is found that the reliability of the galaxy-galaxy lensing signal depends on the number density of galaxies whose distorted shapes can be reliably measured, as well as any additional constraints that can be added to the analysis, for instance, redshifts of the lens galaxies, redshifts of the source galaxies, galaxy type, dynamical constraints, and the presence of larger scale structure like groups or clusters in the vicinity.

All these studies are based on a maximum likelihood analysis of the data sets and the likelihood function is constructed from the ellipticity probability distribution of galaxies. Maximum likelihood methods are preferred to the so-called direct averaging method (which consists of obtaining an average shear field by simply binning up the shear in radial bins from the centre of the lens outwards). The direct averaging method is widely used to constrain galaxy cluster mass profiles. In the case of galaxy-galaxy lensing, since the lensing signal is much smaller than the characteristic noise which corresponds to the width of the intrinsic ellipticity distribution of the galaxies, one has to stack many individual galaxy shear profiles to obtain a signal and to constrain an average galaxy halo population. Such a method is possible when studying isolated field galaxies as in Fisher et al. (2000) study on the SDSS data. The direct averaging method supposes that we are able to isolate a lens in order to study it, which is rarely the case. In fact, galaxy-galaxy lensing is fundamentally a multiple deflection problem. This was first pointed out by BBS in their early work, who found that more than $50 \%$ of their source galaxies should have been lensed by two or more foreground galaxies: the closest lens on the sky to any given source was not necessarily the only lens, nor the strongest one. Moreover, Brainerd (2004b) in an analysis of multiple deflections by the galaxies in the Hubble Deep Field North (HDF) has shown that the probability of multiple deflections exceeds $50 \%$ for source galaxies with a redshift greater than 1 (see also the work by Hoekstra et al. (2005) where they consider isolated galaxies). In many cases it has become clear that it is almost never a unique lens that is responsible for the detected lensing signal and that there are indeed no clean lines of sight (Guzik \& Seljak 2002). Consequently, the problem is best tackled using an "inverse" method, and analysing galaxy-galaxy lensing using maximum likelihood techniques is an example of such a method.

\subsection{Observational results}

The main goal of galaxy-galaxy lensing studies is to obtain constraints on the physical parameters that characterize the dark matter halos of galaxies. A dark matter halo can be described by two parameters: in this work we will use $\sigma_{0}$, the central velocity dispersion, which is related to the depth of the potential well, and $r_{\text {cut }}$, the cut off radius, which is related to the spatial extension of the halo since it defines a change in the slope of the three dimensional mass density profile: below $r_{\text {cut }}$, the profile falls with radius (see Paper I for a complete description of galaxy dark matter halo modeling). It should be noted that a dark matter halo parametrized by $r_{\text {cut }}$ still has a significant amount of mass below $r_{\text {cut }}$ : the mass profile become steeper, but half of the mass is contained below $r_{\text {cut }}$. Thus $r_{\text {cut }}$ can be considered as a half mass radius. More quantitatively, considering a galaxy sized dark matter halo with $\sigma_{0}=220 \mathrm{~km} \mathrm{~s}^{-1}$ and $r_{\text {cut }}=50 \mathrm{kpc}$, we derive that $M\left(R>r_{\text {cut }}\right)=45 \% M_{\text {tot }}$.

In the following, we present galaxy-galaxy lensing results, using $\sigma_{0}$ and $r_{\text {cut }}$ to characterize their properties. In practice, many galaxies have to be stacked in order to reliably detect a signal. As the lenses considered in a given study do not have the same luminosity, they cannot be assigned the same parameters. 


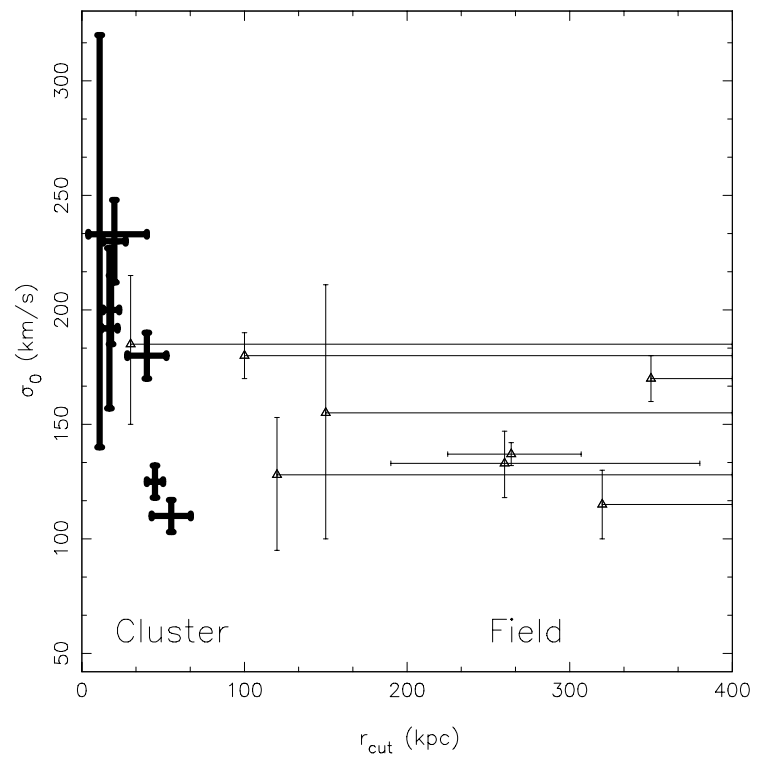

Fig. 1. Comparison between galaxy-galaxy lensing results on cluster galaxies (black) and galaxy-galaxy lensing results on field galaxies (grey). References are given in Sect. 2.2.

The standard approach is to use scaling relations between the different lenses and to derive properties scaled for a given luminosity $L^{*}$ :

$\sigma_{0}=\sigma_{0}^{*}\left(\frac{L}{L^{*}}\right)^{\delta} \quad \& \quad r_{\mathrm{cut}}=r_{\mathrm{cut}}^{*}\left(\frac{L}{L^{*}}\right)^{\alpha}$.

The case $\delta=0.25$ corresponds to the Faber-Jackson/TullyFisher relation. The case $\alpha=0.5$ assumes that the mass to light ratio is constant for all galaxies. These values are the one that are most often used in lensing studies. However, there are other possible scaling relations, and they can be tested with lensing.

We begin with the first non detection of galaxy-galaxy lensing by Tyson et al. (1984). Despite a vast amount of data (about 28000 foreground-background pairs), they were unable to get a galaxy-galaxy lensing signal, mainly because of the poor quality of the data at that time. The first statistically significant detection of galaxy-galaxy lensing is the work by BBS in 1996. They used deep ground-based imaging data $(\simeq 72 \mathrm{sq}$. arcmin) to investigate the orientation of 511 faint background galaxies relative to 439 brighter foreground field galaxies. They claimed a detection of galaxy-galaxy lensing on angular scales between $5^{\prime \prime}$ and $35^{\prime \prime}$ and derived limits on the characteristic parameters of the dark matter halos of $L^{*}$ field galaxies: $\sigma_{0}=155 \pm 56 \mathrm{~km} \mathrm{~s}^{-1}$ and $r_{\text {cut }}>100 h^{-1} \mathrm{kpc}$. Since BBS, there have been different independent detections of galaxy-galaxy lensing by field galaxies. We list them below and illustrate this enumeration in Fig. 1 along with the constraints from these studies on the $\left(\sigma_{0}, r_{\text {cut }}\right)$ plane (for the studies which were able to constrain both parameters, with the values transferred to the cosmology used in this paper). Studies on field galaxies are in grey.

- Griffiths et al. (1996), from the HST Medium Deep Survey, used 1600 foreground objects $(15<I<22)$ and 14000 background objects $(22<I<26)$. They were able to differentiate between spiral and elliptical lenses, and found: $\sigma_{0}=220 \mathrm{~km} \mathrm{~s}^{-1}$ (elliptical) and $\sigma_{0}=155 \mathrm{~km} \mathrm{~s}^{-1}$ (spiral), as well as a constraint on the halo extension: $r \simeq 100 r_{\mathrm{hl}}$, where $r_{\mathrm{hl}}$ is the radius of the luminous component.
- Dell'Antonio \& Tyson (1996), from HDF North, used a simple colour cut to differentiate between lenses and sources: they used 110 lenses and 697 sources, and found $\sigma_{0}=$ $185_{-35}^{+30} \mathrm{~km} \mathrm{~s}^{-1}$ and $r_{\text {cut }}>15 h^{-1} \mathrm{kpc}$.

- Hudson et al. (1998), from HDF North, with photometric redshifts for 208 lenses and 697 sources, found $\sigma_{0}=148 \pm$ $28 \mathrm{~km} \mathrm{~s}^{-1}$ but no constraints on the extension of these halos.

- Ebbels (1998), from the HST Medium Deep Survey, used 22000 objects and a magnitude cut, and found $\sigma_{0}=$ $128_{-34}^{+25} \mathrm{~km} \mathrm{~s}^{-1}$ and $r_{\text {cut }}>120 \mathrm{kpc}$.

- Fisher et al. (2000), from the SDSs data covering 225 sq. degrees, used 28000 bright objects $(16<r<18)$ and 150000 background objects $(18<r<22)$, and found $\sigma_{0}=145-195 \mathrm{~km} \mathrm{~s}^{-1}$ and $r_{\text {cut }}>275 h^{-1} \mathrm{kpc}$.

- Jaunsen (2000), from CFRS fields, with photometric redshifts, found $\sigma_{0}=280 \pm 30 \mathrm{~km} \mathrm{~s}^{-1}$ and no constraints on the extension of these halos.

- McKay et al. (2001), from the sDss data with many more objects than the study by Fisher et al. (2000) and with spectroscopic redshifts for all lenses $\left(3.4 \times 10^{4}\right.$ lenses with $r^{\prime}<17.6$ and $3.6 \times 10^{6}$ sources with $18<r^{\prime}<22$ ), found $\sigma_{0}=100-130 \mathrm{~km} \mathrm{~s}^{-1}$ and $r_{\text {cut }}>230 h^{-1} \mathrm{kpc}$.

- Smith et al. (2001), on the LCRS, used 790 lenses $(R<18)$ and found $\sigma_{0}=116 \pm 14 \mathrm{~km} \mathrm{~s}^{-1}$ and no constraints on the extension of these halos.

- Wilson et al. (2001), studied elliptical galaxies in the redshift range $0.25<z<0.75$. They used 15000 lenses with photometric redshift and 148000 sources $(I>25)$. No evolution in the velocity dispersion with redshift was established. They found $\sigma_{0}=168_{-21}^{+19} \mathrm{~km} \mathrm{~s}^{-1}$ and no constraints on the extension of these halos.

- Hoekstra et al. (2003), from CNOC-2 fields, with a magnitude cut $\left(17.5<R_{\text {bright }}<23\right.$ and $\left.22<R_{\text {faint }}<26\right)$ found $\sigma_{0}=$ $133_{-15}^{+14} \mathrm{~km} \mathrm{~s}^{-1}$ and $r_{\text {cut }}=260_{-73}^{+124} \mathrm{kpc}$.

- Kleinheinrich et al. (2006), applied a maximum likelihood analysis on the COMBO 17 survey (Classifying Objects by Medium-Band Observations in 17 filters) where an accurate estimation of photometric redshift was possible. Considering all lenses, they found $\sigma_{0}=156_{-24}^{+18} \mathrm{~km} \mathrm{~s}^{-1}$, and $r_{\mathrm{vir}}=$ $209_{-32}^{+24} h^{-1} \mathrm{kpc}(1 \sigma$ confidence level $)$. Splitting the lens sample into two subsamples according to the spectral types they found a $2 \sigma$ difference in the velocity dispersion which is larger for early-type galaxies. Moreover, this work provides some constraints on the exponent of the scaling relation on the velocity dispersion (see Eq. (1)), finding $\delta=0.28_{-0.12}^{+0.15}$ for red galaxies, in agreement with the Faber-Jackson relation.

- Hoekstra et al. (2004) used Red Sequence Cluster Survey data (RCS). Lenses were selected as objects as $19.5<R<$ 21 and background objects as $21.5<R<24$. They found $\sigma_{0}=137 \pm 5 \mathrm{~km} \mathrm{~s}^{-1}$ and $r_{\text {cut }}=185_{-28}^{+30} h^{-1} \mathrm{kpc}$.

- Heymans et al. (2006) from HST GEMS data. They constrain the evolution of the virial to stellar mass ratio of galaxies with high stellar mass in the redshift range $0.2 \leq z \leq 0.8$. The estimation of the stellar mass comes from COMBO 17, and the measurements of the virial mass from a galaxy-galaxy lensing analysis. They selected lenses by imposing a high stellar mass cut, and their sample contains a majority of early type galaxies. Space observations provide a number density of 65 source galaxies per square arcminute. They find a virial radius of $\sim 204 h^{-1} \mathrm{kpc}$ and a mean mass to light ratio of $\sim 123 \mathrm{~h}$, in agreement with the findings of Hoekstra et al. (2005). These values can be compared to the one found in the presented work for cluster early type galaxies (Table 1), 
attesting for a strong influence of the environment on the galaxy properties.

- Mandelbaum et al. (2006a) from the SDSs, present constraints on the halo mass of the central galaxy and the fraction of galaxies that are satellites as a function of $r$ band luminosity and stellar mass. Galaxy-galaxy lensing was used to derive virial halo mass, and spectroscopy to derive stellar masses. They looked at the efficiency with which baryons in the halo of the central galaxy have been converted into stars, finding a factor of 2 or more difference in conversion efficiency between typical spirals and ellipticals above stellar mass of $10^{11} M_{\odot}$. They compare some properties of early type galaxies in both low and high-density region, and find both populations to have consistent central halo masses.

\subsection{Discussion}

Examining the studies above, we find that there is considerable variation between data sets and the analysis techniques used by the various authors. The imaging quality, size of the field, and the dichotomy between lenses and sources differ significantly amongst these investigations. Most of them were limited to imaging in a single bandpass hence they used a crude lens/source separation based upon apparent magnitude. Different groups consider different luminosities, make measurements on various scales and therefore an exact comparison between each result is difficult. Moreover, the data are a heterogeneous mix of deep images which were acquired for purposes other than galaxy-galaxy lensing studies.

Despite these differences one has to keep in mind, the implications of these studies for the physical characteristics of the halos of field galaxies are all broadly consistent with one another, which is remarkable, and which is the only robust conclusion that we want to draw about the enumeration of the different detections on field galaxies. Of course, we do not expect all the results to converge to a single set of parameters because each study is different by itself as mentioned before, and the interpretation of the characteristic parameters depends on the morphological type of the galaxy hosted, when most of the study were not able to split their lens sample between early and late type galaxies.

All the different studies do fit reasonable central velocity dispersions, i.e. they are consistent with results inferred from more traditional techniques such as rotation curves. In the case of galaxy halos in the field no clear edge is detected to the mass distribution even on scales of the order of a few hundred kpc (McKay et al. 2001; Fisher et al. 2000). Only two published studies to date by Hoekstra et al. (2003) and Hoekstra et al. (2004) have been able to put an upper bound on the characteristic extension of a field halo at about $290_{-82}^{+139} h^{-1} \mathrm{kpc}$ and $185_{-28}^{+30} h^{-1} \mathrm{kpc}$. Besides these large values do not impose a stringent constraint for typical galaxy mass distributions since at these radii the galaxy density is only a few times above the mean density of the Universe.

These results on field galaxies are in rather good agreement with studies based on satellite dynamics (Zaritsky et al. 1993, 1997; Prada et al. 2003; Brainerd 2004a) where the idea is to use a satellite galaxy as a test particle to probe the gravitational potential of a brighter host galaxy that is considered to be more massive. This method is feasible for isolated field galaxies, and the authors have found the extension of dark matter halos of isolated galaxies to be larger than $200 \mathrm{kpc}$. The samples of host and satellites in current redshift surveys are becoming large enough to be used to study the dark matter halos of the host galaxies: this is emerging as a powerful technique that is entirely complementary to galaxy-galaxy lensing.

We can say that the last ten years since the first detection of galaxy-galaxy lensing have been "experimental" in the sense that these early studies have demonstrated convincingly that galaxygalaxy lensing, though challenging to detect, is a viable technique by which the dark matter distribution on scales of individual galaxies can be investigated. Now that the technique has been proved, galaxy-galaxy lensing shows great promise on getting interesting statistical constraints on galaxy physics. At present the constraints obtained are not very strong, but the preliminary results are very encouraging. In particular, the following investigations are currently pursued:

- halo parameter determination, mass measurement and the $M / L$ ratio: evolution with redshift and influence of the local environment, any evolutionary effects are investigated

- relation between the baryonic and dark matter components to provide constraints for models of galaxy formation

- deviation from spherical symmetry: there are both observational and theoretical arguments in favor of flattened halos, and galaxy-galaxy lensing can provide constraints on the mean flattening of the dark matter halos of field galaxies (see e.g. Brainerd \& Wright 2001). Hoekstra et al. (2004) presented a weak lensing detection of the flattening of galaxy dark matter halos, with an ellipticity $\sim 0.2$, implying that the halos are aligned with the light distribution. Recently, Mandelbaum et al. (2006b) detected an ellipticity from the SDSS data set, which appears to be mildly inconsistent with the detection reported by Hoekstra et al. (2004). Note however that these two works used different data and methodology, making a direct comparison difficult. Moreover, it is worth mentioning that probing the shape of dark matter halos is still a difficult measurement because one tries to measure an azimuthal variation of the galaxy-galaxy lensing signal which is itself challenging to detect

- morphological dependence of the halo potential: dynamical studies suggest that the depth of the potential wells of earlytype $L^{*}$ galaxies is deeper than those of late-type $L^{*}$ galaxies. The fact that early-type galaxies are more often observed as acting as strong lenses than late-type galaxies (Kochanek et al. 2000) reinforces this idea. Some studies have been able to differentiate between spiral and elliptical lenses (Griffiths et al. 1996; McKay et al. 2001; Guzik \& Seljak 2002; Kleinheinrich et al. 2006; Hoekstra et al. 2005), and all these studies have found their elliptical galaxy sample to produce stronger galaxy-galaxy lensing signal than their spiral galaxy sample. McKay et al. (2001) converted their galaxy-galaxy lensing signal to an aperture mass in an aperture radius of $260 h^{-1} \mathrm{kpc}$ and found it to be a factor 2.7 larger for ellipticals than for spirals. Guzik \& Seljak (2002) in their work on the SDSS data, also found that the virial mass $M^{*}$ of an $L^{*}$ galaxy varies significantly with galaxy morphology, with $M^{*}$ being lower for late types relative to early types (up to a factor 10 in the $u^{\prime}$ band). It is interesting to find a similar result by using satellite dynamics: Brainerd (2004a) computed the velocity dispersion profile for the satellites of host galaxies in the Two Degree Field Galaxy Redshift Survey and in the $\Lambda$ CDM GIF simulation. She found the velocity dispersion profile to have a substantially higher amplitude and steeper slope for satellite of early-type hosts than it does for satellites of late-type hosts 
- the scaling of the total galaxy mass with luminosity, including any strong evolution of these relations with redshift: McKay et al. (2001) split their lens sample with luminosity, and found the shear signal to be strongly dependent on the luminosity: the more luminous the lenses, the stronger the shear they produce, hence the more massive they are. These scaling laws have been confirmed by an independent dynamical method applied on the same data set (McKay et al. 2002). Hoekstra et al. (2004) also provide a constraint on the scaling relation between the $B$-band luminosity and the velocity dispersion, and found a relation that is in very good agreement with the Tully-Fisher relation. Moreover, Hoekstra et al. (2005) considered isolated galaxies from the RCS: they split their sample into 7 luminosity bands and measure the mean tangential shear signal out to 2 arcmin from the lens. They find that the strength of the lensing signal increases with the luminosity of the lens. As a consequence, the virial mass is found to be an increasing function of the luminosity, with a slope of $\sim 1.5$ in $B, V$ and $R$ bands.

- the truncation of the dark matter halos during the infall of galaxies into cluster (see below).

- the bias of light compared to mass by studying the galaxymass correlation function (Seljak et al. 2005).

- the nature of the dark matter: Natarajan et al. (2002a) have shown that constraints on the extent of the mass distribution around galaxies in the rich cluster Abell 2218 obtained from combining strong and weak lensing observations are consistent with the predictions which assume that the dominant mass component (dark matter) in these halos is collisionless. A strongly interacting (fluid-like) dark matter is ruled out at a confidence level of more than $5 \sigma$.

- comparison between the virial and the stellar mass and constraints on the star formation efficiency (Heymans et al. 2006; Hoekstra et al. 2005; Guzik \& Seljak 2002; Mandelbaum et al. 2006a).

Future prospects are very promising: in particular, the exploitation of the cosmos survey is ongoing. This survey is a 2 sq. degree imaging survey with the HST; it will contain about $10^{6}$ galaxies and about 35000 spectra of galaxies to be measured with the VIMOs instrument on the VLT. Moreover, the multi wavelength observations will assign secure photometric redshifts for all objects. As demonstrated by Kleinheinrich et al. (2005), knowledge of the lens redshifts is very important in any galaxy-galaxy lensing study. Applying galaxy-galaxy lensing techniques to the full cosmos data will give interesting results on galaxy physics, and will relate the galaxy properties to their redshift and the local environment. Furthermore, the next generation of space telescopes will allow probing deeper in the Universe by combining wide field and very high quality data from space. From the ground, ongoing or future surveys are also very promising for galaxy-galaxy lensing studies (the CFHTLS, the second generation of RCS survey, as well as the KIlo Degree Survey (KIDS)).

\subsection{Galaxy-galaxy lensing through clusters}

Galaxy-galaxy lensing has been used successfully to map substructure in massive lensing clusters (Natarajan et al. 1998; Geiger \& Schneider 1999; Natarajan et al. 2002a,b, and the work presented in this paper). Figure 2 shows the different galaxygalaxy lensing results on cluster galaxies. Analyses on cluster galaxies all used HST data for their investigations. The study of Geiger \& Schneider (1999) on galaxy cluster C10939+4713

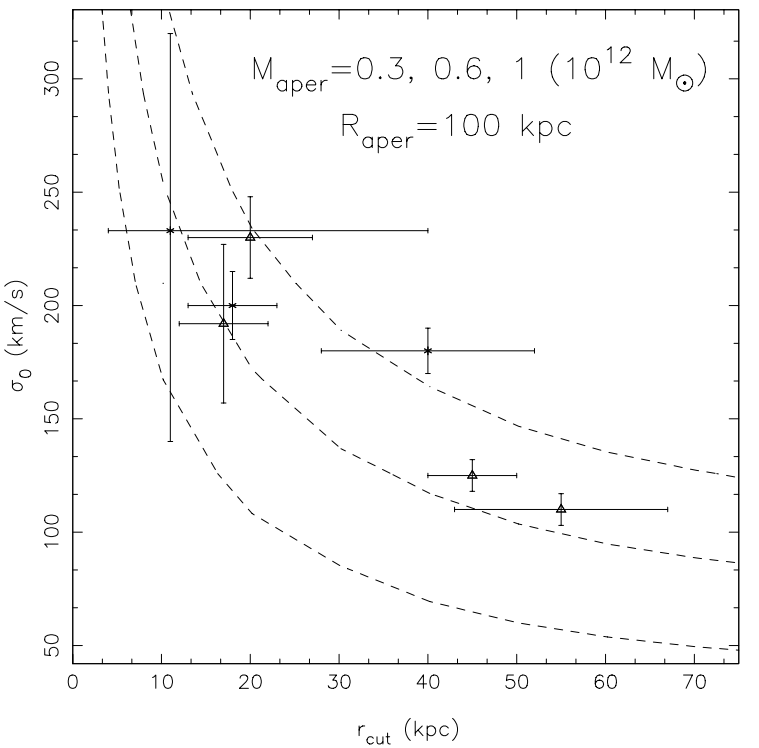

Fig. 2. Results obtained on cluster galaxies from galaxy-galaxy lensing analysis. Along the dotted lines, the mass within a projected radius $R_{\text {aper }}=100 \mathrm{kpc}$ is constant, equal to the value indicated on the plot.

led to a detection of galaxy-galaxy lensing, but the field appeared to be too small to allow strong conclusions to be drawn about the mass distributions of the cluster galaxies. Studies led by Natarajan et al. included strong constraints from observation of multiple images systems which boost the convergence of the likelihood, which is why their error bars are tighter than in the study by Geiger \& Schneider.

From Fig. 1, a clear trend can be seen: dark matter halos in cluster are significantly more compact compared to halos around field galaxies of equivalent luminosity. This is a landmark observational result from galaxy-galaxy lensing studies, that was expected from theoretical considerations and numerical simulations: when clustering, galaxies experience strong tidal stripping from the cluster potential, and they loose part of their dark matter halo, feeding the global cluster dark matter halo itself. Moreover, Natarajan et al. (2002b) have considered five galaxy clusters which span a wide range of redshifts $(0.18<z<0.58)$ and they find that not only are the dark matter halos truncated in dense environment, the proper length of the truncation radius increases with the redshift as expected from tidal stripping scenarios (Ghigna et al. 1998). However, given the small field of the HST data used there are likely to be limitations from systematics. The clusters used are a very heterogeneous sample (they span a wide range in redshift, richness, mass, X-ray luminosity) and possibly an increased proportion of contaminating field galaxies for the higher redshift clusters.

\section{Observations and cataloging}

\subsection{Data}

The data used in the presented work were taken at the CFHT with the CFH12K camera through the $B, R$ and $I$ filters. A detailed description of the data acquisition and reduction can be found in Czoske (2002). For a brief outline see Bardeau et al. (2005). No further details will be given in this paper.

The average seeing of the observations as estimated from the $F W H M$ of stars is $0.8^{\prime \prime}$ in the $R$ band. The average limiting magnitude in the $R$ band is equal to 26.2. 


\subsection{From images to catalogues}

For a detailed description of the object detection see Bardeau et al. (2005). Here we just give a brief outline of the different steps involved in the analysis of the reduced and calibrated images. A crucial step is to estimate the Point Spread Function (PSF) and its variation in each region of the images. The PSF measures the response of the entire optical system (atmosphere + telescope optics) to a point source. In our case, stars provide the calibrating point source, thus the shapes of stars detected in the images provide our estimate of the PSF. The shape of a star includes an isotropic component mainly due to atmospheric seeing, as well as an anisotropic component caused, for example, by small irregularities in the telescope guiding. The isotropic component of the PSF leads to a circularization of the images of small galaxies and thus reduces the amplitude of the measured shear. The anisotropic PSF component introduces a systematic component in galaxy ellipticities and thus causes a spurious shear measurement if not corrected (Kaiser et al. 1995).

The images were processed through various software routines in order to extract the quantities we are interested in: the position of each galaxy, the shape parameters and their magnitudes. The first step is to construct a photometric catalogue for each individual image. In order to get the object positions and magnitudes, we have used SEXTRACTOR (Bertin \& Arnouts 1996). The second step of the analysis is to extract a star catalogue from the full catalogue which will be used to estimate the local PSF. We selected stars and cleaned the resulting catalogue as described in Bardeau et al. (2005). In order to measure the shapes of the stars, we used the IM2SHAPE software developed by Bridle et al. (2001). At this stage, we have a map of the PSF distribution over the entire field. The third step is to compute the galaxy catalogues that will be used in the weak lensing analysis. Galaxies are selected from the photometric catalogues according to the criterion described in Bardeau et al. (2005). To measure the shapes of galaxies, we first linearly interpolate the local PSF at each galaxy position by averaging the shapes of the five closest stars. This number of stars is found to be large enough to locally interpolate the PSF, whereas choosing a much larger number would over-smooth the PSF characteristics. IM2SHAPE then computes the intrinsic shapes of galaxies by convolving a galaxy model with the interpolated local PSF, and determines which one is the most likely by minimizing residuals. In the end, IM2SHAPE's output gives a most likely model for the fitted galaxy characterized by its position, size, ellipticity and orientation, and errors on all of these quantities.

Finally, a master catalogue is produced which matches the objects detected in the three filters and which contains colour indices built from aperture magnitudes in 16 pixels (3.28") diameter apertures. This catalogue is used to plot the colour-magnitude diagrams from which the sequence of elliptical is identified and extracted. Bardeau et al. (2005) used the full objects catalogues in their weak lensing analysis. In this paper, we use only objects detected in all three bands and with reliable shape information. Reliable shapes refers to objects for which the error on the ellipticity is small. If $e=e_{1}+\mathrm{i} e_{2}$ is the complex ellipticity, we impose the errors on these parameters to be $\operatorname{err}\left(e_{1}\right)<0.1$ and $\operatorname{err}\left(e_{2}\right)<0.1$. Moreover, the shape parameters used in the final maximum likelihood analysis are the ones derived from the $R$ band, as the data quality in terms of seeing and source density is superior compared to the other bands. These objects with three colours constitute the basis of our galaxygalaxy lensing analysis and we undertake a photometric study of these objects to derive a redshift estimation for each object.

\section{Bayesian photometric redshifts}

Getting photometric redshifts with three bands is quite challenging but possible and reliable for certain redshift ranges which are well constrained by the filters we have. Adding a prior probability allows us to get better constraints than we would have without any assumptions. The method implemented here has been developed by Benitez (1999). We will first verify the calibration of the magnitude, and then show the results of a theoretical study we undertook in order to quantify the kind of information we can derive from the data at hand. Then we will verify that the photometric redshift determination is correct.

\subsection{Verification of the magnitude calibration}

Before using magnitudes in the analysis, we need to verify that they are well calibrated. From the colour-magnitude diagram, we are able to define the most luminous elliptical galaxies of the cluster on the red cluster sequence (about 50 objects). We then compare their colours to the Coleman et al. (CWW) templates, which are found to be a better comparison set to our galaxies than the Bruzual \& Charlot (2003) elliptical template. We selected the most luminous objects because the CWW template corresponds to a metallicity close to solar metallicity. Moreover, we choose the CWW template because it comes from observations of elliptical galaxies in the local universe, and we can reasonably assume that there is little evolution between the redshift of the cluster sample $(z \sim 0.2)$ and the present day. The magnitude calibration appeared to be correct.

\subsection{Theoretical analysis}

Using the HYPERZ (Bolzonella et al. 2000) templates, we are able to simulate a catalogue with known $z_{\text {model }}$ and the corresponding $B, R$ and $I$ colours. These colours are then fed back to HYPERZ in order to derive an estimate of the photometric redshift $z_{\text {phot }}$ (to compute the magnitudes, a noise is estimated as a function of the apparent magnitude as explained in Bolzonella et al. 2000). We can delineate "good" and "bad" regions with respect to e.g. the criterion proposed by Schneider \& Rix (1997): a region is considered as a good one if the points do verify the following constraint: $0.5<z_{\text {phot }} / z_{\text {model }}<1.5$ :

- from $z=0$ to $z=0.5$, the constraints derived from HYPERZ are bad; only $30 \%$ of the objects satisfy the criterion;

- from $z=0.5$ to $z \sim 1$, we have a good region, with $60 \%$ of the objects that satisfy the criterion;

- from $z \sim 1$ to $z=3.7,73 \%$ of the objects do satisfy the criterion;

- from $z \sim 3.7$ to $z=5,89 \%$ of the objects do satisfy the criterion, note that with increasing redshift, this criterion becomes less difficult to satisfy.

We now restrict our study to the redshift range $z=0$ to $z=1.5$ because this is the range where we are likely to have the majority of background objects:

- by comparison to the HDF (Bardeau et al. 2005), we found that the mean redshift of the background population is $z \sim 1$

- moreover, Fig. 3 shows the $I$ magnitude distribution for the whole catalogue of Abell 1763 and for the cleaned catalogue, i.e. when we restrict ourselves to the objects with a good measurement of the shape parameters. We see that the magnitude limit for the clean catalogue is about 24 . Our catalogue can be compared to the VIMOs "deep field" 

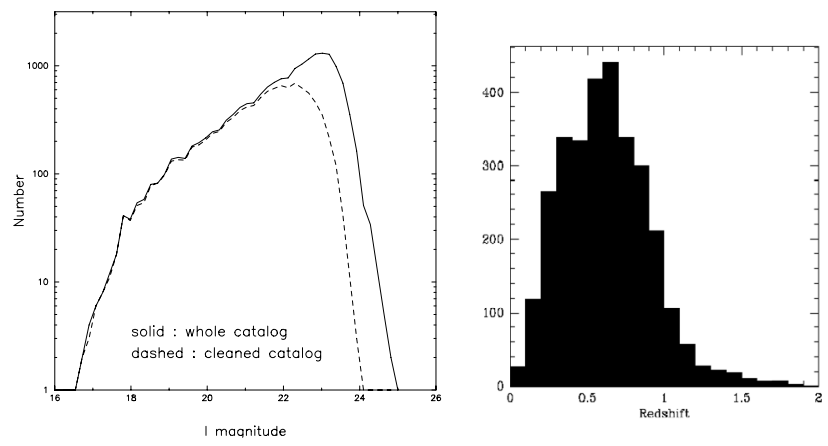

Fig. 3. Left: I magnitude distribution for the whole catalogue (solid line) and for the cleaned catalogue (dashed line). Right: redshift distribution from VIMOS VLT Deep Survey galaxies that verify $I$ magnitude $<24$.

(Le Fèvre et al. 2003), for which a redshift distribution is known: they use a sample of galaxies $I<24$ and from the redshift distribution for this sample,we see (Fig. 3) that most of the objects are located at $z<1.4$, with a tail out to $z=2$ (5\% contamination above 1.4$)$. By comparison, we deduce that most of our objects are located at redshifts lower than 1.4 .

The second step for the analysis is to perform a Bayesian photometric study for these simulated objects. The idea is to add a prior probability which is not used by HYPERZ and in which we are confident. This extra information will add constraints to get the redshift distribution of an object. We will use as a prior the luminosity function. The final redshift assigned to a galaxy is determined by combining the information coming from the HYPERZ probability distribution with the prior probability. Basically, adding this prior allows us to get rid of some degeneracies in the redshift probability distribution coming from HYPERZ: using only three filters, the spectral energy distribution of a given galaxy is not well constrained, thus the redshift probability distribution sometimes exhibits two solution: a moderate and a high redshift one. The prior then "kills" some unlikely high redshift solution, for example when a relatively bright galaxy has a redshift probability distribution with a moderate redshift solution $(z \sim 0.5-1)$ and a high redshift solution $(z \sim 3)$. We can delineate "good" and "bad" regions as before:

- from $z=0$ to $z=0.5$, only $40 \%$ of the objects do satisfy the Schneider \& Rix (1997) criterion. It is better than when not adding the prior, but still the redshift is poorly constrained in this range;

- from $z=0.5$ to $z=1.5,98 \%$ of the objects do satisfy the criterion.

We see that having a Bayesian approach to the problem by adding a prior probability does improve the redshift determination significantly in the range where it was already reliable before adding this prior. Note however that this theoretical analysis is idealized in the sense that it is based on synthetic galaxy templates and does not include any contamination by stars. The redshift determination is quite reliable for the background population $(z>0.5)$. These objects will be used as the background sample in the galaxy-galaxy lensing analysis. On the other hand, the redshift of the lenses is not well constrained by our filters. In particular, the cluster ellipticals are assigned a Bayesian redshift between 0.35 and 0.45 , systematically overestimating their redshift. So we decided to extract the elliptical galaxies from a colour magnitude diagram and assign them a redshift equal to the redshift of the cluster. These objects will be the lenses in the following analysis. We are aware that we will loose some lenses

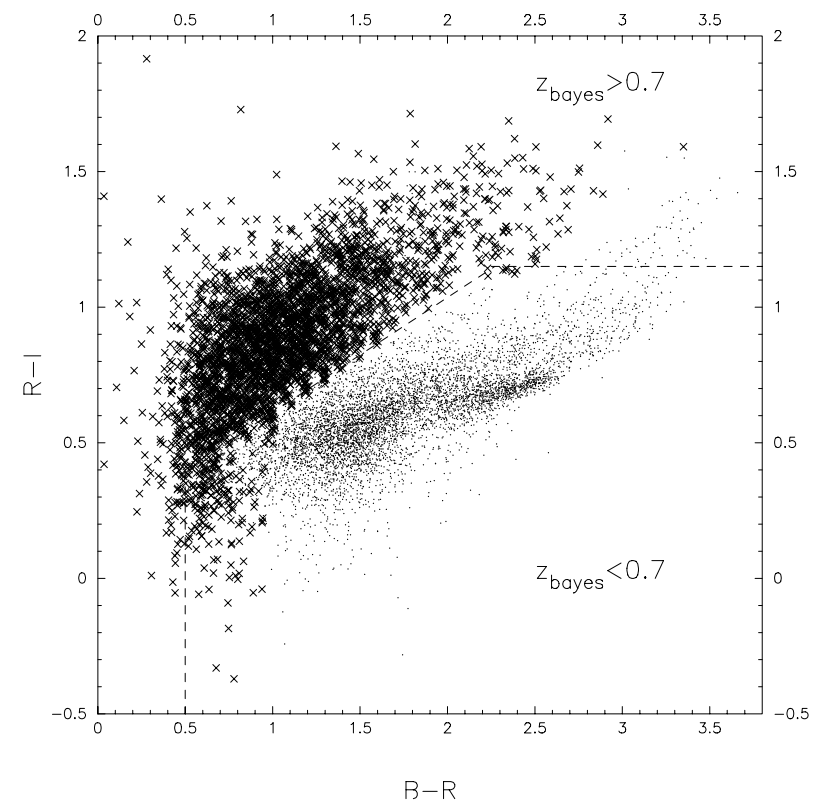

Fig. 4. Objects of our Abell 1763 catalogue with $z_{\text {bayes }}>0.7$ (crosses) and with $z_{\text {bayes }}<0.7$ (dots). The line represents the simple colour-cut used in the DEEP2 survey to select objects with $z>0.7$ and $z<0.7$.

in doing so, but the advantage is to have the right redshift for the cluster elliptical galaxies which are supposed to dominate the galaxy-galaxy lensing signal.

\subsection{Reliability of the Bayesian photometric redshift determination: comparison to the DEEP2 survey}

To verify the reliability of the Bayesian photometric redshift estimation, we compare with the DEEP2 redshift survey (Coil et al. 2004a). In the DEEP2 galaxy redshift survey, they use a simple colour-cut designed to select galaxies at $z>0.7: B-R<$ $2.35 \times(R-I)-0.45, R-I>1.15$ or $B-R<0.5$. As discussed in Davis et al. (2003), this colour-cut has proven effective: it results in a sample with $\sim 90 \%$ of the objects at $z>0.7$, missing only $\sim 5 \%$ of the $z>0.7$ galaxies. We check to see where our objects with $z_{\text {bayes }}>0.7$ and $z_{\text {bayes }}<0.7$ fall in a colour-colour diagram, with respect to this colour-cut. Figure 4 shows the results. The dashed line represents the colour-cut: objects at $z>0.7$ are supposed to be above the regions defined by these three dashed lines according to the DEEP 2 colour-cut. The points represent our objects for which we have estimated $z_{\text {bayes }}<0.7$. The crosses represent our objects for which we have estimated $z_{\text {bayes }}>0.7$. We can see from this plot that our estimation of the redshift agrees well with the colour-cut. The next section gives an outline of the methodology used in this work.

\section{Methodology}

Full details on the methodology are given in Paper I. Here we briefly explain the method: we introduce the parameters used to describe the galaxy mass profile. Then we outline the maximum likelihood analysis used to derive constraints on dark matter halos of galaxies.

\subsection{Modeling the mass distribution of galaxies}

Beginning with the three dimensional density distribution $\rho(r)$ that fully characterizes a dark matter halo for our purposes, we 
project it onto the lens plane to get the two dimensional potential, $\phi(R)$. The related projected surface mass density $\Sigma$ is then given by:

$4 \pi \mathrm{G} \Sigma(R)=\nabla^{2} \phi(R)$

and the two-dimensional projected mass inside radius $R$ (the aperture radius $R_{\text {aper }}$ ) is defined as follows:

$M_{\text {aper }}(R)=2 \pi \int_{0}^{R} \Sigma(r) r \mathrm{~d} r$.

To model dark matter halos, we use the two components PseudoIsothermal Elliptical Mass Distribution (PIEMD, Kassiola \& Kovner 1993), which is a more physically motivated mass profile than the isothermal sphere profile (SIS) but sharing the same profile slope at intermediate radius. The density distribution for this model is given by:

$\rho(r)=\frac{\rho_{0}}{\left(1+r^{2} / r_{\text {core }}^{2}\right)\left(1+r^{2} / r_{\text {cut }}^{2}\right)}$

with the core radius $r_{\text {core }}$ of the order of $0.1^{\prime \prime}$, and a truncation radius $r_{\text {cut }}$. In the centre, $\rho \sim \rho_{0} /\left(1+r^{2} / r_{\text {core }}^{2}\right)$ which describes a core with central density $\rho_{0}$. The transition region ( $\left.r_{\text {core }}<r<r_{\text {cut }}\right)$ is isothermal, with $\rho \sim r^{-2}$. In the outer parts, the density falls off as $\rho \sim r^{-4}$, as is usually required for models of elliptical galaxies. This mass distribution is described by a central velocity dispersion $\sigma_{0}$ related to $\rho_{0}$ for a circular potential by the following relation:

$\rho_{0}=\frac{\sigma_{0}^{2}}{2 \pi \mathrm{G}}\left(\frac{r_{\text {cut }}+r_{\text {core }}}{r_{\text {core }}^{2} r_{\text {cut }}}\right)$.

It is easy to show that for a vanishing core radius, the density profile obtained becomes identical to the density profile used by BBS for modeling galaxy-galaxy lensing. Since many authors are using the same mass profile used by BBS in their galaxy-galaxy lensing studies, it allows for easy comparison with our results.

\subsection{Cluster description}

We describe the cluster component as a large scale smooth component (see Paper I for details). This cluster component is put in by hand and we use the results found by Bardeau (2004) on the same data set as parameters.

\subsection{Maximum likelihood analysis}

The details of the method have been given in Paper I. Here we give a brief outline of the method.

Once we have the image catalogue, we process it through a numerical code that retrieves the input parameters of the lenses using a maximum likelihood method as proposed by Schneider $\&$ Rix (1997). For each image (i), given a mass model for the foreground lenses galaxies (e.g. $\sigma_{0}, r$ ), we can evaluate the amplification matrix $a_{i}$ as a contribution of all the foreground galaxies $j ; z_{j}<z_{i}$ that lies within a circle of inner radius $R_{\min }$, and outer radius $R_{\max }$ and of centre the position of the image $(i)$ :

$$
a_{i}\left(\sigma_{0}, r\right)=\sum_{z_{j}<z_{i}} a_{i j}\left(\sigma_{0}, r\right) .
$$

Given the observed ellipticity $\boldsymbol{\varepsilon}_{\mathrm{obs}}^{i}$ and the associated amplification matrix $a_{i}$, we are able to retrieve the intrinsic ellipticity $\varepsilon_{i}^{\mathrm{s}}$ of the source before lensing:

$\boldsymbol{\varepsilon}_{i}^{\mathrm{s}}=F\left(\boldsymbol{\varepsilon}_{\mathrm{obs}}^{i}, a_{i}\left(\sigma_{0}, r\right)\right)=\boldsymbol{\varepsilon}_{i}^{\mathrm{s}}\left(\sigma_{0}, r\right)$.

In order to assign a likelihood to the parameters used to describe the lense galaxies, we use $P^{\mathrm{s}}$, the ellipticity probability distribution in the absence of lensing. Doing that for each image of the catalogue, we construct the likelihood function:

$\mathcal{L}\left(\sigma_{0}, r\right)=\prod_{i} P^{\mathrm{s}}\left(\boldsymbol{\varepsilon}_{i}^{\mathrm{s}}\right)$

which is a function of the parameters used to define the mass models of the lenses. For each pair of parameters, we can compute a likelihood. The larger this function, the more likely the parameters used to describe the lenses. See Paper I for a discussion on the convergence properties of this likelihood function.

As exposed in detail in Paper I, we found that galaxy-galaxy lensing studies are first sensitive to the mass enclosed within a given radius; this gave us the idea to re-parameterize the problem in terms of more physical quantities: the aperture mass calculated in an aperture radius. Instead of fitting the deformations in the $\left(\sigma_{0}, r_{\text {cut }}\right)$ plane, we can fit them directly in the $\left(M_{\text {aper }}, R_{\text {aper }}\right)$ plane. We have:

$\mathcal{L}=\mathcal{L}\left(\sigma_{0}, r_{\text {cut }}\right) \quad \& \quad M_{\text {aper }}=M_{\text {aper }}\left(R_{\text {aper }}, \sigma_{0}, r_{\text {cut }}\right)$

so we can write:

$\sigma_{0}=\sigma_{0}\left(M_{\text {aper }}, R_{\text {aper }}, r_{\text {cut }}\right)$

the likelihood function then becomes:

$\mathcal{L}\left(M_{\text {aper }}, R_{\text {aper }}, r_{\text {cut }}\right)$

and by summing over $r_{\text {cut }}$, we obtain:

$\mathcal{L}^{\prime}=\sum_{r_{\text {cut }}} \mathcal{L}\left(M_{\text {aper }}, R_{\text {aper }}, r_{\text {cut }}\right)$

this means that:

$\mathcal{L}^{\prime}=\mathcal{L}^{\prime}\left(M_{\text {aper }}, R_{\text {aper }}\right)$

The new likelihood function is then a function of the aperture mass and the aperture radius. Note that $M_{\text {aper }}$ and $R_{\text {aper }}$ are not independent parameters, which explains the shape of the likelihood contours in the $\left(M_{\text {aper }}, R_{\text {aper }}\right)$ plane: they remain open along the $R_{\text {aper }}$ axis.

In Paper I we tested extensively the maximum likelihood method on simulated data defined to match observations to study the accuracy with which input parameters for mass distributions for galaxies can be extracted. We showed that the two standard parameters that characterise galaxy halo models, the central velocity dispersion and the truncation radius can be retrieved reliably from the maximum likelihood analysis and that the proposed re-parameterization allows us to put strong constraints on the aperture mass of a galaxy halo (with less than $10 \%$ error). Thus we are confident in applying this method to the data set presented in Sects. 3 and 4.

\section{Results of the galaxy-galaxy lensing study}

In this section, we present the results we have for the elliptical galaxies in the field of the different clusters studied in this paper. In the following plots, the likelihood contours are the $1 \sigma, 2 \sigma$ and $3 \sigma$ confidence level contours. 


\subsection{Null tests}

In order to examine the validity of the galaxy-galaxy lensing signal, we generated a non physical catalogue as follows:

- the orientation angle $\theta$ for the galaxies is randomly assigned;

- the position of galaxies that verify $z_{\text {bayes }}>0.5$ is randomly assigned;

- the position of the foreground cluster elliptical galaxies is randomly assigned.

In each case, the likelihood function does not exhibit any significant maximum.

\subsection{Catalogues}

The catalogues used in the maximum likelihood analysis are formed by the objects with the following characteristics:

- 3 colored objects (it means objects detected in the three bands: $B, R, I$ )

- shape parameters coming from the $R$ band which appeared to be the less noisy one;

- errors on the estimation of the ellipticity lower than 0.1 ;

- redshift assigned at the redshift of the cluster for ellipticals galaxies, Bayesian photometric redshifts for the background population $(z>0.5)$.

The average number of lenses in the considered catalogues is $\sim 700$, and the average number of background sources is $\sim 7500$.

This catalogue is the input to the maximum likelihood code. We use the scaling relations given in Sect. 2.2, with $\delta=0.25$ and $\alpha=0.5$. From theoretical considerations, the $R_{\max }$ parameter should be of the order of 100" (see Paper I).

\subsection{Results}

Results are presented in Fig. 5. We fit the deformations with a PIEMD profile, in the $\left(\sigma_{0}, r_{\text {cut }}\right)$ plane and in the $\left(M_{\text {aper }}, R_{\text {aper }}\right)$ plane. Table 1 summarizes the results we obtained. The main results are the following: (i) we fit reasonable values for the velocity dispersions, around $200 \mathrm{~km} \mathrm{~s}^{-1}$. This is reasonable in the sense that it is comparable to values inferred using more traditional methods (rotation curves, X-ray observations, satellite dynamics); (ii) we find dark matter halos to be very compact compared to field galaxies of equivalent luminosity: considering all cluster galaxies halos, an upper limit on the truncation radius is set at $50 \mathrm{kpc}$ (PIEMD results on Abell 383), when the truncation radius inferred on field galaxies is found to be larger than a few hundreds of kpc (see Sect. 2 and Fig. 1). As mentioned in Sect. 2.2, the truncation radius is related to the extension of the halo. We can say that cluster galaxies are more compact than field galaxies because the slope of their mass profile steepens earlier, thus the corresponding mass profile reaches a low density value earlier. As a consequence, dark matter halo of cluster galaxies are less extended than they are in the case of field galaxies.

Galaxy cluster Abell 1689 was also studied as part of the galaxy cluster sample. We found similar constraints for galaxy halos living in this cluster, but the significance of the detection is below the $1 \sigma$ level, so we do not add this detection in this paper.

It should also be pointed out that detections for the different cluster galaxy dark matter halos are comparable to one another; this is due to the fact that these different clusters all have similar physical properties and constitute a very homogeneous sample.
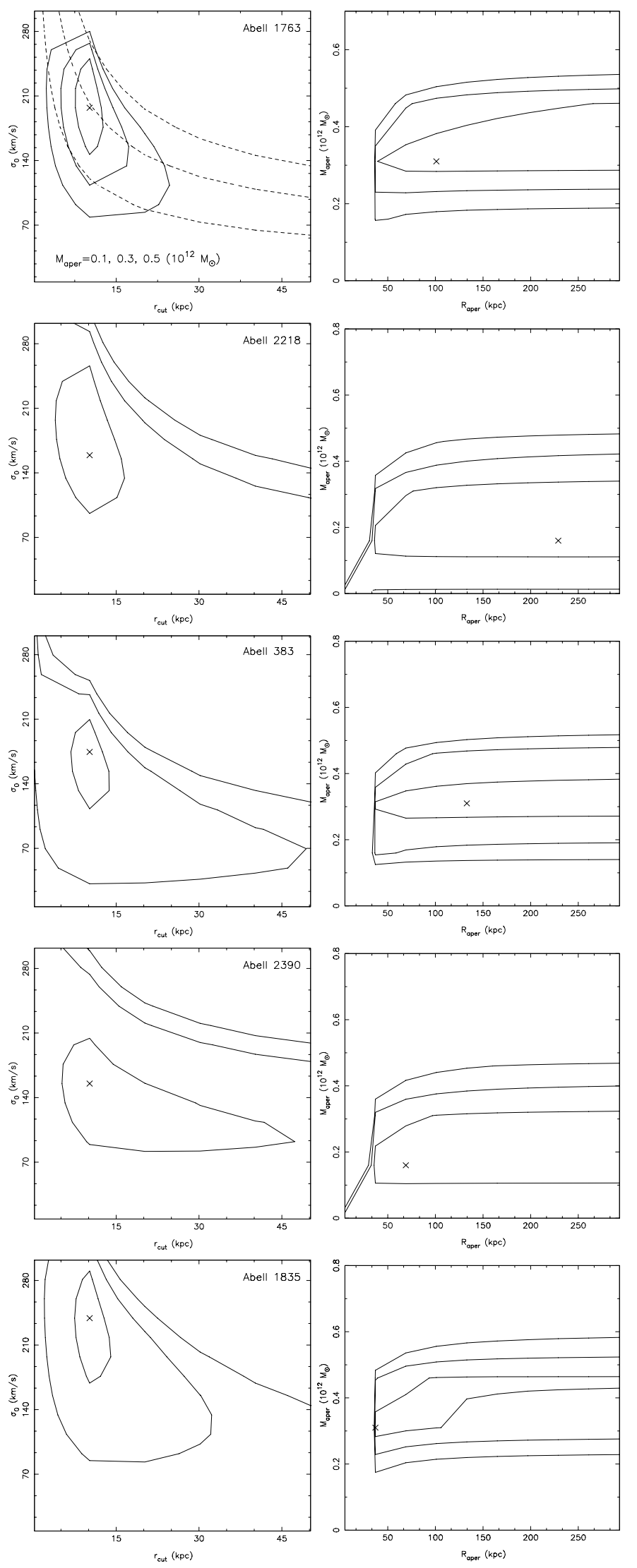

Fig. 5. Results of the galaxy-galaxy lensing analysis in the $\left(\sigma_{0}, r_{\text {cut }}\right)$ plane (left $)$ and in the $\left(M_{\text {aper }}, R_{\text {aper }}\right)$ plane. From top to bottom, Abell 1763, Abell 2218, Abell 383, Abell 2390, Abell 1835. In the case of Abell 1763, along the dotted lines in the $\left(\sigma_{0}, r_{\text {cut }}\right)$ plane, the aperture mass computed in an aperture radius of $100 \mathrm{kpc}$ is kept constant equal to the value indicated on the plot. 
Table 1. Summary of the detections, for a $L^{*}$ luminosity. The mass corresponds to the total mass computed with a PIEMD profile, and luminosity comes from the $R$ band. Here $\sigma$ corresponds to the confidence level of the detection.

\begin{tabular}{cccc}
\hline \hline Cluster & $\sigma_{0}^{*}, \mathrm{~km} \mathrm{~s}^{-1}($ PIEMD) & $r_{\text {cut }}^{*}$, kpc $($ PIEMD $)$ & $(M / L)^{*}$ \\
\hline A1763 & $200_{-115}^{+70}(3 \sigma)$ & $\leq 25(3 \sigma)$ & $19_{-6}^{+16}(3 \sigma)$ \\
A1835 & $240_{-159}^{+81}(2 \sigma)$ & $\leq 32(2 \sigma)$ & $20_{-6}^{+18}(3 \sigma)$ \\
A2218 & $200_{-64}^{+96}(1 \sigma)$ & $\leq 18(1 \sigma)$ & $13_{-12}^{+10}(2 \sigma)$ \\
A383 & $175_{-143}^{+66}(2 \sigma)$ & $\leq 50(2 \sigma)$ & $20_{-10}^{+13}(3 \sigma)$ \\
A2390 & $155_{-75}^{+50}(1 \sigma)$ & $\leq 47(1 \sigma)$ & $10_{-4}^{+21}(1 \sigma)$ \\
\hline
\end{tabular}

\subsection{Comparison with other results on cluster galaxies}

Figure 6 shows a comparison of our results (in black) with results on cluster galaxies from Natarajan et al. and from Geiger $\&$ Schneider (in grey). There is a good agreement between the different studies, though the data sets as well as the analysis are quite different: as mentioned before studies by Natarajan et al. and Geiger \& Schneider are based on HST data and therefore they probe central cluster galaxies. Natarajan et al. used the constraints derived from the observations of multiple images, whereas Geiger \& Schneider did not include strong lensing constraints in their analysis. Our results are averaged on a cluster galaxy population from the centre to $\sim 2 \mathrm{Mpc}$, thus we probe the whole centre of the cluster as well as part of the transition region.

The results presented in this work can also can be compared to the constraints found by Smith et al. (2005) on the cluster galaxies of a galaxy cluster sample that contain the galaxy clusters we study in this work. Smith et al. (2005) presented an analysis of $10 \mathrm{X}$-ray luminous galaxy clusters based on HST observations. Therefore they probe the inner part of the galaxy cluster. From the observation of multiply imaged systems, they modeled the mass distribution in the cluster cores $(R<500 \mathrm{kpc})$. In order to reproduce the location of multiple images detected on the image, each cluster model comprised of a number of parameterized mass components which account for mass distributed on both cluster and galaxy scales. Therefore, their study also gives some constraints on the characteristic parameters of the cluster galaxies. Using a PIEMD profile, they found: $\sigma_{0}=180 \pm 20 \mathrm{~km} \mathrm{~s}^{-1}$ and $r_{\text {cut }}=23 \mathrm{kpc}$, in very good agreement with the results presented here.

The strong lensing modeling of galaxy cluster Abell 1689 from HST ACS observations by Halkola et al. (2006) also provides evidence for cluster galaxies to be significantly stripped, with a cut radius of $24 \mathrm{kpc}$.

The work by Mandelbaum et al. (2006a) also gives some insight on the extension of the dark matter halos: for early type galaxies living in high density regions, they probe the extension of the dark matter halos by searching for a depression in the lensing signal relative to that for field galaxies on $50-100 h^{-1} \mathrm{kpc}$ scales. A large amount of tidal stripping in clusters would cause a depression in the lensing signal on scales below the virial radius. No such depression was found by the authors, ruling out scenarios that have most satellites strongly stripped. This result seems to be in disagreement with the one presented in this paper and with early results of galaxy-galaxy lensing in clusters. It should be noted that in order to look for a depression in the lensing signal, one needs to use a direct averaging method, which is not well suited for studying cluster galaxies that are very close one to each other compared to field galaxies (see

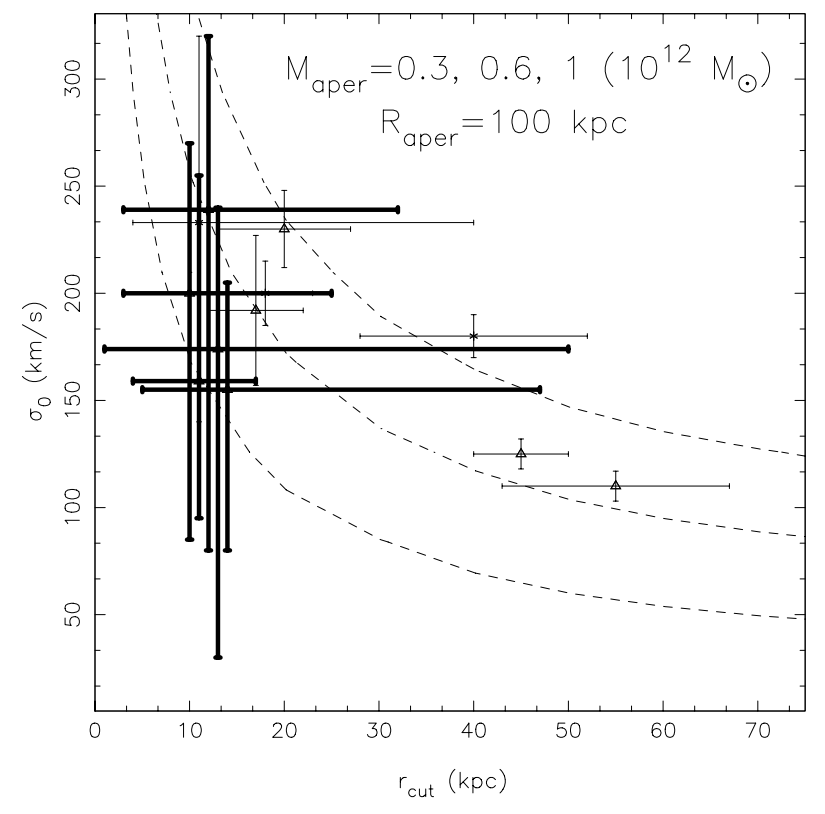

Fig. 6. Comparison of our results (black) with the results from Natarajan et al. and Geiger \& Schneider (grey). Note that a comparison is possible keeping in mind that the different data sets and the methods used are different.

Sect. 2.1). Moreover, as mentioned before, a dark matter halo with $r_{\text {cut }} \sim 50 \mathrm{kpc}$ (as found in this work) still has a significant amount of mass below $50 \mathrm{kpc}$ and will generate a non negligible lensing signal below $50 \mathrm{kpc}$. Moreover, it is worth mentioning the fact that we do present some constraints for galaxies inhabiting very massive clusters where tidal stripping is expected to be more efficient, thus our results are biased towards very high density environments. Therefore the different results are not easy to compare and may not be in disagreement.

\section{Discussion and conclusion}

The results presented here come from wide field ground based data. As a consequence, we probe the cluster galaxy population out to a larger radius than previous studies $(\sim 2 \mathrm{Mpc})$. This means that our results are averaged over a galaxy population located all the way from the centre of the cluster to the transition region of the cluster. Our study confirms the fact that galaxy halos in clusters are significantly more compact compared to halos around field galaxies of equivalent luminosity.

The results presented in this work are in very good agreement with numerical simulations. A detailed comparison with realistic numerical simulations will be presented in a forthcoming publication (see also recent work by Natarajan et al. 2006). The theoretical expectation is that the global tidal field of a massive, dense cluster potential well should be strong enough to truncate the dark matter halos of galaxies that traverse the cluster core. As mentioned before, this expectation has been observationally confirmed by several independent previous studies. Early numerical work (see e.g Merritt 1983; Malumuth \& Richstone 1984) noticed that a large fraction of the mass initially attached to galaxies in the central megaparsec is stripped. Bullock et al. (2001) found that halos in dense environments are more truncated than their isolated counterparts of the same virial mass. Avila-Reese et al. $(1999,2005)$ found that halos in cluster regions are more concentrated than isolated halos. $N$-body simulations of cluster formation and evolution (Ghigna et al. 1998; 
Ghigna et al. 2000) find that the dominant interactions are between the global cluster tidal field and individual galaxies after $z=2$. The cluster tidal field significantly strips galaxy halos. As previously noted, we probe in this paper lens galaxies located all the way from the centre of the cluster to $\sim 2 \mathrm{Mpc}$. This galaxy population is bound to the cluster and it is reasonable to think that it has experienced the cluster potential at least once: the characteristic time for a galaxy to cross a cluster is about $10^{9}$ years, and the age of a galaxy cluster that formed at $z=1$ is about $10^{10}$ years.

To conclude, we presented the first galaxy-galaxy lensing results to date that probe cluster galaxies from a ground based survey. This study has confirmed the fact that galaxy halos in clusters are significantly less massive and more compact compared to galaxy halos around field galaxies of equivalent luminosity. Moreover, this confirmation is based on the analysis of 5 massive clusters lenses whose properties are close to each other, hence the confirmation we provide is a strong one since it relies on a homogeneous sample.

Acknowledgements. The Dark Cosmology Centre is funded by the Danish National Research Foundation. M.L. wishes to acknowledge Roser Pellò for help in using HYPERZ, in understanding photometric redshift determination and for useful discussions and encouragements. M.L. also thanks many people for carefully reading this paper and for constructive comments, in particular: Alexie Leauthaud, Árdís Elíasdóttir, Gary Mamon, Jens Hjorth and Jesper SommerLarsen. P.N. acknowledges gratefully support from NASA via HST grant HSTGO-09722.06-A. Argelander-Institut für Astronomie is founded by merging of the Institut für Astrophysik und Extraterrestrische Forschung, the Sternwarte, and the Radioastronomisches Institut der Universität Bonn. IRS and GPS acknowledge support from the Royal Society.

\section{References}

Avila-Reese, V., Firmani, C., Klypin, A., \& Kravtsov, A. V. 1999, MNRAS, 310, 527

Avila-Reese, V., Colín, P., Gottlöber, S., et al. 2005, ApJ, 634, 51

Bardeau, S. 2004, Ph.D. Thesis, Université Paul Sabatier

Bardeau, S., Kneib, J.-P., Czoske, O., et al. 2005, A\&A, 434, 433

Benitez, N. 1999, in Edited by Ray Weymann, Lisa Storrie-Lombardi, Marcin Sawicki, and Robert Brunner. ISBN: 1-58381-017-X (1999), ASP Conf. Ser., 191, 31 [arXiv: astro-ph/9811189]

Bertin, E., \& Arnouts, S. 1996, A\&AS, 117, 393

Bolzonella, M., Miralles, J. M., \& Pelló, R. 2000, A\&A, 363, 476

Brainerd, T. G., Blandford, R. D., \& Smail, I. 1996, ApJ, 466, 623

Brainerd, T. G., \& Wright, C. O. 2001, PASP, submitted

[arXiv:astro-ph/0006281]

Brainerd, T. G. 2004, ApJ, submitted [arXiv: astro-ph/0409381]

Brainerd, T. G. 2004, to be published in the proceedings of IAU Symp., 225, The Impact of Gravitational Lensing on Cosmology, ed. Y. Mellier, \& G. Meylan [arXiv: astro-ph/0409374]

Bridle, S., Gull, S., Bardeau, S., \& Kneib, J.-P. 2001, in Proc. of the Yale Cosmology Workshop: The Shapes of Galaxies and their Dark Halos, ed. N. P. (World Scientific)

Bruzual, G., \& Charlot, S. 2003, MNRAS, 344, 1000

Bullock, J. S., Kolatt, T. S., Sigad, Y., et al. 2001, MNRAS, 321, 559

Coil, A. L., Davis, M., Madgwick, D. S., et al. 2004, ApJ, 609, 525

Coleman, D. G., Wu, C. C., \& Weedman, D. W. 1980, ApJS, 43, 393

Czoske, O. 2002, Ph.D. Thesis, Université Paul Sabatier
Davis, M., Faber, S. M., Newman, J., et al. 2003, Proc. SPIE, 4834, 161 Dell'Antonio, I. P., \& Tyson, J. A. 1996, ApJ, 473, L17

Ebbels, T. 1998, Ph.D. Thesis, University of Cambridge

Ebeling, H., Voges, W., Bohringer, H., et al. 1996, MNRAS, 281, 799

Faber, S. M., \& Jackson, R. E. 1976, ApJ, 204, 668

Fassnacht, C. D., Blandford, R. D., Cohen, J. G., et al. 1999, ApJ, 117, 658

Fisher, P., McKay, T. A., Sheldon, E., et al. (the sDss collaboration) 2000, ApJ, 120,1198

Geiger, B., \& Schneider, P. 1998, MNRAS, 295, 497

Geiger, B., \& Schneider, P. 1999, MNRAS, 302, 118

Ghigna, S., Moore, B., Governato, F., et al. 1998, MNRAS, 300, 146

Ghigna, S., Moore, B., Governato, F., Quinn, T., \& Stadel, J. 2000, ApJ, 544, 616

Griffiths, R. E., Casertano, S., Im, M., \& Ratnatunga, K. U. 1996, MNRAS, 282, 1159

Guzik, J., \& Seljak, U. 2002, MNRAS, 335, 311

Halkola, A., Seitz, S., \& Pannella, M. 2006, MNRAS, 372, 1425

Heymans, C., Bell, E. F., Rix, H.-W., et al. 2006, MNRAS, 371, L60

Hoekstra, H., Franx, M., Kuijken, K., Carlberg, R. G., \& Yee, H. K. C. 2003, MNRAS, 340, 609

Hoekstra, H., Yee, H. K. C., \& Gladders, M. D. 2004, ApJ, 606, 67

Hoekstra, H., Hsieh, B. C., Yee, H. K. C., et al. 2005, ApJ, 635, 73

Hudson, M. J., Gwyn, S. D. J., Dahle, H., \& Kaiser, N. 1998, ApJ, 503, 531

Jaunsen, A. O. 2000, Ph.D. Thesis, University of Oslo

Kaiser, N., Squires, G., \& Broadhurst, T. 1995, ApJ, 449, 460

Kassiola, A., \& Kovner, I. 1993, ApJ, 417, 450

Kleinheinrich, M., Rix, H. W., Erben, T., Schneider, P., et al. 2005, A\&A, 439, 513

Kleinheinrich, M., Schneider, P., Rix, H.-W., et al. 2006, A\&A, 455, 441

Kneib, J.-P. 1993, Ph.D. Thesis, Université Paul Sabatier

Kneib, J.-P., Ellis, R. S., Smail, I., Couch, W. J., \& Sharples, R. M. 1996, ApJ, 471,643

Kochaneck, C. S., Falco, E. E., Impey, C. D., et al. 2000, ApJ, 543, 131

Le Fèvre, O., Vettolani, G., Maccagni, D., et al. 2003, in Proceedings of IAU Symp. 216, Maps of the Cosmos, Sydney, July 2003, ed. M. Colless, \& L. Staveley-Smith [arXiv: astro-ph/0311475]

Limousin, M., Kneib, J.-P., \& Natarajan, P. 2005, MNRAS, 356, 309

Malumuth, E. M., \& Richstone, D. O. 1984, ApJ, 276, 413

Mandelbaum, R., Seljak, U., Kauffmann, G., Hirata, C. M., Brinkmann, J., et al. 2006a, MNRAS, 368, 715

Mandelbaum, R., Hirata, C. M., Broderick, T., Seljak, U., Brinkmann, J., et al. 2006b, MNRAS, 370, 1008

Mc Kay, T. A., Sheldon, E. S., Racusin, J., et al. 2001, ApJ, submitted [arXiv: astro-ph/0108013]

Mc Kay, T. A., Sheldon, E. S., Johnston, D., et al. 2002, ApJ, 571, L85

Merritt, D. 1983, ApJ, 264, 24

Natarajan, P., \& Kneib, J.-P. 1997, MNRAS, 287, 833

Natarajan, P., Kneib, J.-P., Smail, I., \& Ellis, R. 1998, ApJ, 499, 600

Natarajan, P., Loeb, A., Kneib, J.-P., \& Smail, I. 2002a, ApJ, 580, L17

Natarajan, P., Kneib, J.-P., \& Smail, I. 2002b, ApJ, 580, L11

Natarajan, P., De Lucia, G., \& Springel, V. 2006, MNRAS, submitted [arXiv: astro-ph/0604414]

Phillips, P. M., Norbury, M. A., Koopmans, L. V. E., et al. 2000, MNRAS, 319, L7

Prada, F., Vitvitska, M., Klypin, A., et al. 2003, ApJ, 598, 260

Refregier, A. 2003, ARA\&A, 41, 645

Schneider, P., \& Rix, H. W. 1997, ApJ, 474, 25

Seljak, U., et al. 2005, Phys. Rev., 127, 2544

Smith, D., Bernstein, G. M., Fisher, P., \& Jarvis, M. 2001 ApJ, 551, 643

Smith, G. P., Kneib, J.-P., Smail, I., et al. 2005, MNRAS, 359, 417

Tully, R. B., \& Fisher, J. R. 1977, A\&A, 54, 661

Tyson, J. A., Valdes, F., Jarvis, J. F., \& Mills, A. P. 1984, ApJ, 281, L59

Wilson, G., Kaiser, N., Luppino, G., \& Cowie, L. L. 2001, ApJ, 555, 572

Zaritsky, D., Smith, R., Frenk, C., \& White, S. D. M. 1993, ApJ, 405, 464

Zaritsky, D., Smith, R., Frenk, C., \& White, S. D. M. 1997, ApJ, 478, 39 